\title{
3D strain map of axially loaded mouse tibia: a numerical analysis validated by experimental measurements
}

\author{
Vincent A. Stadelmann, Jean Hocké, Jensen Verhelle, Vincent Forster, Francesco Merlini, \\ Alexandre Terrier and Dominique P. Pioletti* \\ Laboratory of Biomechanical Orthopedics EPFL-HOSR, Institute of Translational Biomechanics, Ecole Polytechnique \\ Fédérale de Lausanne, Lausanne, Switzerland
}

(Received 14 December 2007; final version received 2 May 2008)

\begin{abstract}
A combined experimental/numerical study was performed to calculate the 3D octahedral shear strain map in a mouse tibia loaded axially. This study is motivated by the fact that the bone remodelling analysis, in this in vivo mouse model should be performed at the zone of highest mechanical stimulus to maximise the measured effects. Accordingly, it is proposed that quantification of bone remodelling should be performed at the tibial crest and at the distal diaphysis. The numerical model could also be used to furnish a more subtle analysis as a precise correlation between local strain and local biological response can be obtained with the experimentally validated numerical model.
\end{abstract}

Keywords: mechanical loading; bone remodelling; strain quantification; tibia

\section{Introduction}

In vivo mechanical loading models have been developed to study mechano-transduction, which is the process of biological response to a mechanical stimulus. In particular, for bone, the biological response to an applied external mechanical stimulus is generally quantified by morphological imaging (micro-computed tomography, scanning electron microscopy), histology or biomechanical tests (three or four point bending).

Rubin et al. (1996) were pioneers in this field showing that cortical bone mass and size of the turkey ulna was regulated by mechanical stimulations (Rubin and Lanyon 1984). Since this work, different studies have been performed confirming the responsiveness of bone to mechanical stimulus. For example, Chow et al. (1993) showed that mechanical loading is a determinant for the physiological behaviour of cancellous bone in a rat vertebrae model (Chambers et al. 1993). More recently, non-invasive models of mechanical loading were adapted to the challenging dimensions of mice bones. Cyclic four-point loading were applied on mouse tibia and the results highlighted the difference in response between different breeds of mice (Akhter et al. 1998). Tibial axial loading was used to study site-specific remodelling response to mechanical stimuli (De Souza et al. 2005; Fritton et al. 2005) and the loading effect on bone healing (Gardner et al. 2006).

In vivo loading experiments require a precise control of the mechanical stimulus to correctly interpret the measured changes of the bone structure. It is likely that the most relevant structural changes of the loaded bone will be observed at the maximally stimulated locations. Therefore, to optimise the significance of a study, the choice of the location where histological or histomorphological imaging is to be processed should be defined in function of the spatial distribution of the mechanical stimulus.

The most accessible measure of the mechanical stimulus is the strain. The strain variable has indeed been used in many different bone remodelling models (Carter 1987; Huiskes et al. 1987) In this study, we want to estimate the 3D strain map in an axial loading model of the mouse tibia.

The mouse tibia is neither homogenous nor axisymmetric. Therefore, the maximum strain location is not known a priori when an external load is applied. In order to assess the spatial distribution and intensity of strain in an axially loaded mouse tibia, we designed a study based on three steps. The first step was to experimentally measure the strain occurring at three different locations on a mouse tibia under different axial loads. In the second step, a numerical biomechanical model of a mouse tibia was built and validated with the experimental data. In the third step, the numerical model was used to extrapolate the strain distribution over the whole tibial geometry and to determine the 3D octahedral shear strain map and the highest strain locations.

\section{Materials and methods}

In this section, we will first describe the experimental procedure of measuring the strain at three different locations on the mouse tibia, followed by a description

*Corresponding author. Email: dominique.pioletti@epfl.ch

ISSN 1025-5842 print/ISSN 1476-8259 online

(C) 2009 Taylor \& Francis

DOI: $10.1080 / 10255840802178053$

http://www.informaworld.com 


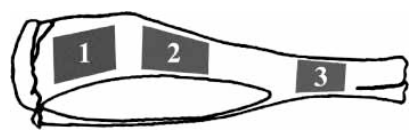

Figure 1. Definition of the regions of interest on the mouse tibia. Zone 1, proximal tibia, presents a flat and regular surface. It is the widest area of the tibia; therefore the easiest location to place a gauge. Zone 2 begins at the tibial crest and ends $1 \mathrm{~mm}$ before the junction with the fibula. This zone is relatively flat but narrower than Zone 1, therefore, the strain gauge is harder to place. Zone 3, distal tibia, is narrow and curved. It is the hardest surface to fix a gauge.

of the numerical model developed to quantify the strain on the whole mouse tibia.

\subsection{Animals}

C57BL6 male mice were acclimated to our facility for 3 weeks. They were maintained under standard no barrier conditions and had access to mouse chow and water ad libitum. The local ethics committee on animal care approved all animal procedures (Protocol\#1920).

\subsection{Strain measures}

Nine mice were sacrificed at the age of $15 \pm 3$ weeks and the tibias were immediately extracted. Soft tissues were removed and the tibias were cleaned with acetone. The tibias were separated randomly into three groups of six corresponding to three zones of tests on the tibia; Zone 1, antero-proximal; Zone 2, antero tibial crest; Zone 3, postero-distal (Figure 1). Each tibia received a single element foil strain gauge (EA-06-015LA-120, Vishay Micro-Measurements, Raleigh, NC, USA) aligned with the long axis bond to the bone surface with cyanoacrylate (Rapid Mix 72771, Forbo CTU, Schönenwerd, Switzerland). The gauge was connected to a tension amplifier and digital recorder (DAQ NI9215, National Instruments,
Switzerland). To verify the linkage between the strain gauge and the bone surface, two tibias were scanned by micro computed tomography $(\mu \mathrm{CT})$ at $9 \mu \mathrm{m}$ pixel size. The images showed that the gauge location corresponded to the target and that no space was left between the gauge and the bone surface (Figure 2).

\subsection{Calibration of the setup}

A strain gauge was placed at the center of an aluminum beam of dimensions $3 \times 10 \times 350 \mathrm{~mm}$ and connected to the electronics. One end of the beam was fixed and controlled, while loads were applied on the opposite end. The resulting tension was recorded for each load. To obtain the deformation-tension relationship, the beam deformation theory was applied to calculate the exact deformation at the center of the beam for each load.

\subsection{Mechanical loading}

A compression machine was developed to apply controlled compression cycles on mouse tibias, based on a previously published work (De Souza et al. 2005; Fritton et al. 2005). Custom moulded pads were placed on the axes to apply the compression on the bone ends. The tibia was then placed on the stimulation machine between the moving pad on the proximal-side and the fixed pad on the distal-side (Figure 3).

To maintain the initial position of the tibia, a pre-load of $0.2 \mathrm{~N}$ was applied before the dynamic compression. The compression waveform was composed of square like cycles at $2 \mathrm{~Hz}$ of frequency and amplitude from $1 \mathrm{~N}$ minimum force during $0.25 \mathrm{~s}$ followed by maximal force during $0.25 \mathrm{~s}$. The maximal force increased from 1 to $10 \mathrm{~N}$ by steps of $1 \mathrm{~N}$ every 20 cycles (Figure 4). Because of the natural curvature of the tibia, this simple axial loading induced combined compressive and bending strains.
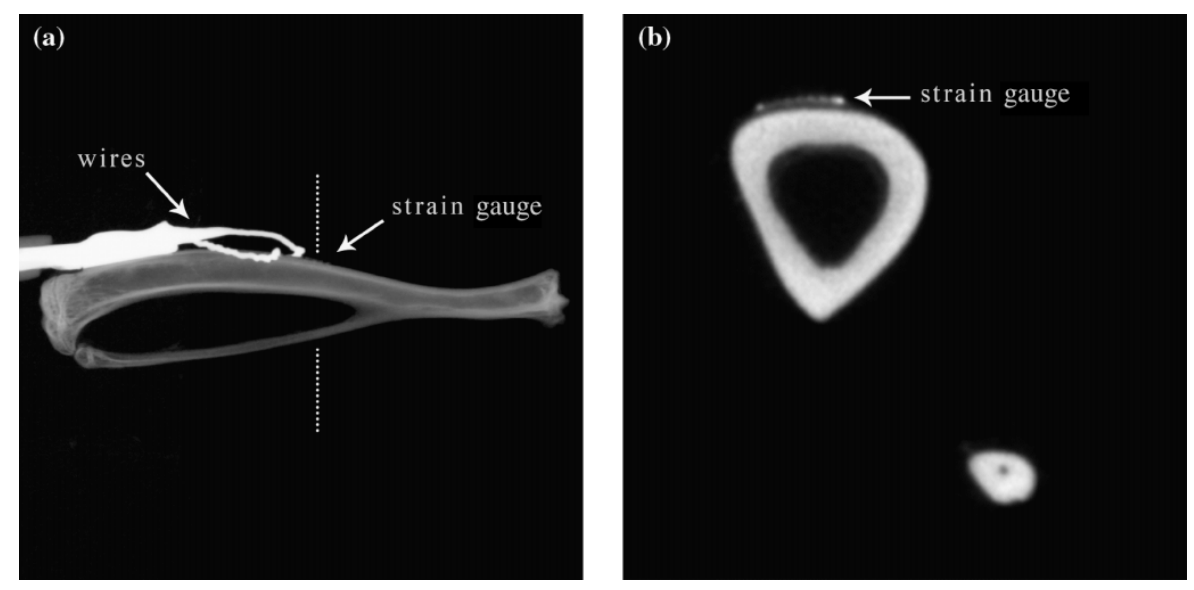

Figure 2. (a) Side-view of a $\mu \mathrm{CT}$ scan of a tibia with a strain gauge placed at Zone 2 and connecting wires. (b) Profile view along dotted line showing the strain gauge linkage to the bone follows the contour of bone surface. 


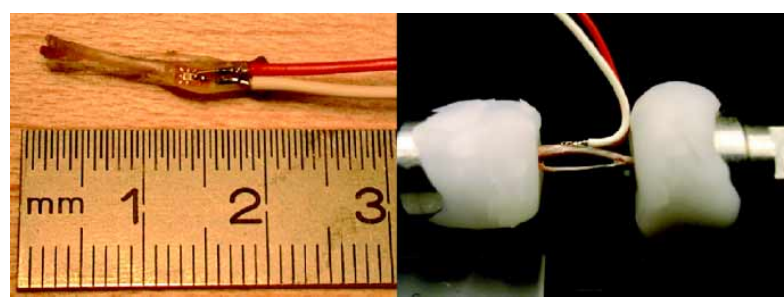

Figure 3. (a) Tibia with strain gauge fixed in Zone 2. (b) Tibia placed in the loading machine with strain gauge in Zone 2 .

\subsection{Numerical model}

\subsubsection{Geometry}

The geometry of a fresh specimen of mouse tibia was reconstructed from $\mu \mathrm{CT}$ scan data ( $\mu$ CT40, Scanco Medical AG, Switzerland) at a $9 \mu \mathrm{m}$ resolution. The axial reconstructed images were then imported in AMIRA (Mercury Computer Systems, MA, USA) for the segmentation of the tibial bone volume. Finally, a geometric model based on parametric surfaces was built from the AMIRA data using Geomagic (Geomagic, Inc., NC, USA).

\subsubsection{D FE model}

The geometric model of the tibia was then imported into Abaqus (Simulia, Providence, RI, USA). A total number of 26,000 3D (20-node quadratic brick, reduced integration) elements were used to mesh the tibia.

\subsubsection{Material properties}

The density of the cancellous and cortical bone can vary greatly in the tibia. To incorporate this parameter, the Hounsfield number was correlated to the bone density. The Young's modulus was then estimated using the equation derived by Rho et al. (1995) for a longitudinal compressed human tibia bone.

\subsubsection{Boundary conditions}

Each of the articular end surfaces of the tibia were rigidly connected to a point placed at the center of the surface.

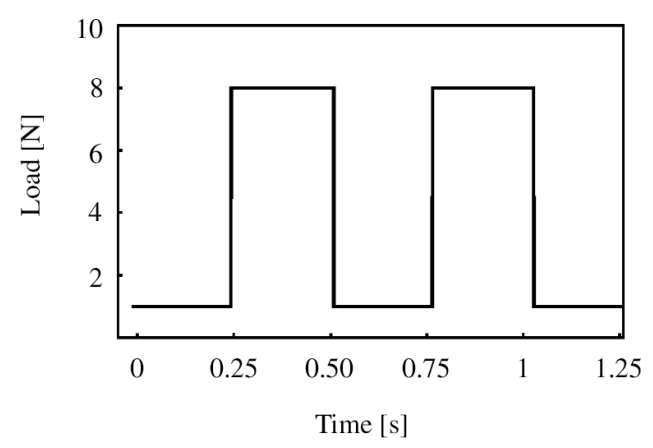

Figure 4. Loading signal waveform with a frequency of $2 \mathrm{~Hz}$ and maximum intensity at $8 \mathrm{~N}$ for two consecutive loading cycles.
The distal point was completely fixed, while the proximal point was free to translate in the axial direction. An axial compressive force of $8 \mathrm{~N}$ was applied on the proximal point.

\subsubsection{Measurements}

Strains were calculated in the direction of the experimental strain gauge, within the three locations (surface of bone) of the strain gauges. An average and standard deviation of the calculated strain was then obtained for each location.

\section{Results}

\subsection{Experimental strain}

At loading application, the strain has a peak of $\sim 10 \%$ of the total strain and then drops to its plateau value until load is retrieved (Figure 5(a)). The following measurements were recorded at plateau values.

The force-strain relationship was established by successively increasing loading cycles between 1 and $10 \mathrm{~N}$ for each zone. The force-strain relationships are linear with $R^{2}>0.95$ for each zone (Figure 5(b)).

For the $8 \mathrm{~N}$ loading, the experimental values of the strain were $440 \pm 31 \mu \varepsilon$ at Zone $1,1337 \pm 100 \mu \varepsilon$ at Zone 2 and $444 \pm 81 \mu \varepsilon$ at Zone 3. The strain values were significantly higher in Zone 2 compared to Zones 1 and 3.

\subsection{Numerical model validation}

For $8 \mathrm{~N}$ loading, the numerical values of the strain were $472 \pm 225 \mu \varepsilon$ at Zone $1,1320 \pm 372 \mu \varepsilon$ at Zone 2 and $420 \pm 127 \mu \varepsilon$ at Zone 3. These results correspond to experimental measurements with an error smaller than 10\% (Figure 6).

\subsection{Extrapolation to the entire tibia, highest strain location}

The octahedral shear strain distribution was calculated numerically for five different locations: proximal tibia, proximal-diaphysis, tibial-crest diaphysis, midshaft and distal tibia (Figure 7). Mean octahedral shear strain was calculated for each zone on the antero and the postero side (Table 1). The maximal octahedral shear strain was found at the postero-tibial crest $(1800 \pm 40 \mu \varepsilon)$ and the anterodistal tibia $(1940 \pm 30 \mu \varepsilon)$.

\section{Discussion}

Although bone adaptation to mechanical environment has been observed for decades, the mechanisms underlying the perception of the mechanical stimulus by the cell and the 
(a)

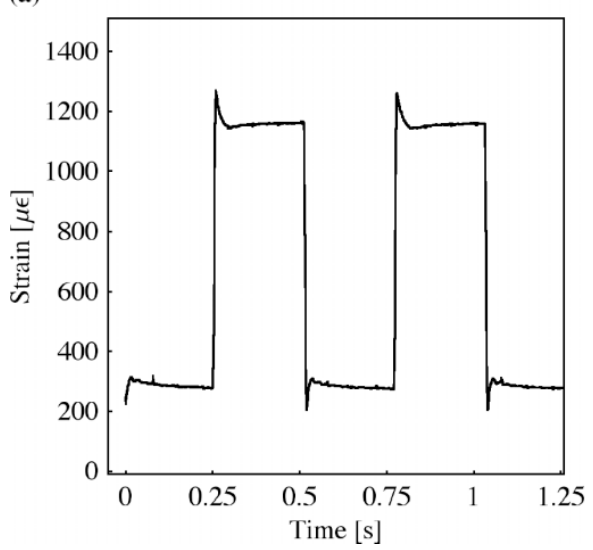

(b)

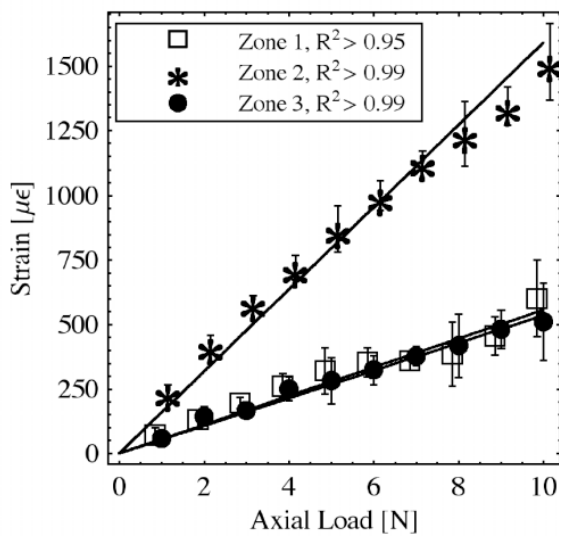

Figure 5. (a) Strain records at Zone 2 for a dynamic load of $8 \mathrm{~N}$ for two consecutive loading cycles. (b) Force-strain experimental relations measured at three zones with the respective linear regressions and $R^{2}$ values.

biological pathways of the cell response are still far from being completely understood.

The in vivo mechanical stimulation of mice tibias combined with histology and histomorphometry $(\mu \mathrm{CT})$ is certainly one of the most promising techniques to gain further understanding in the bone remodelling response to mechanical stimulation. However, histology and $\mu \mathrm{CT}$ are both very time-consuming techniques, and therefore these techniques are generally used only at few locations of the tibia (generally the midshaft, proximal and distal sections), regardless of the loading state.

The goal of this study was to estimate the 3D map of the octahedral shear strain distribution within the tibia of a mouse under specific axial loading that corresponds to our experimental setup. Octahedral shear strain intensity is a good approximation of the mechanical stimulus that cells

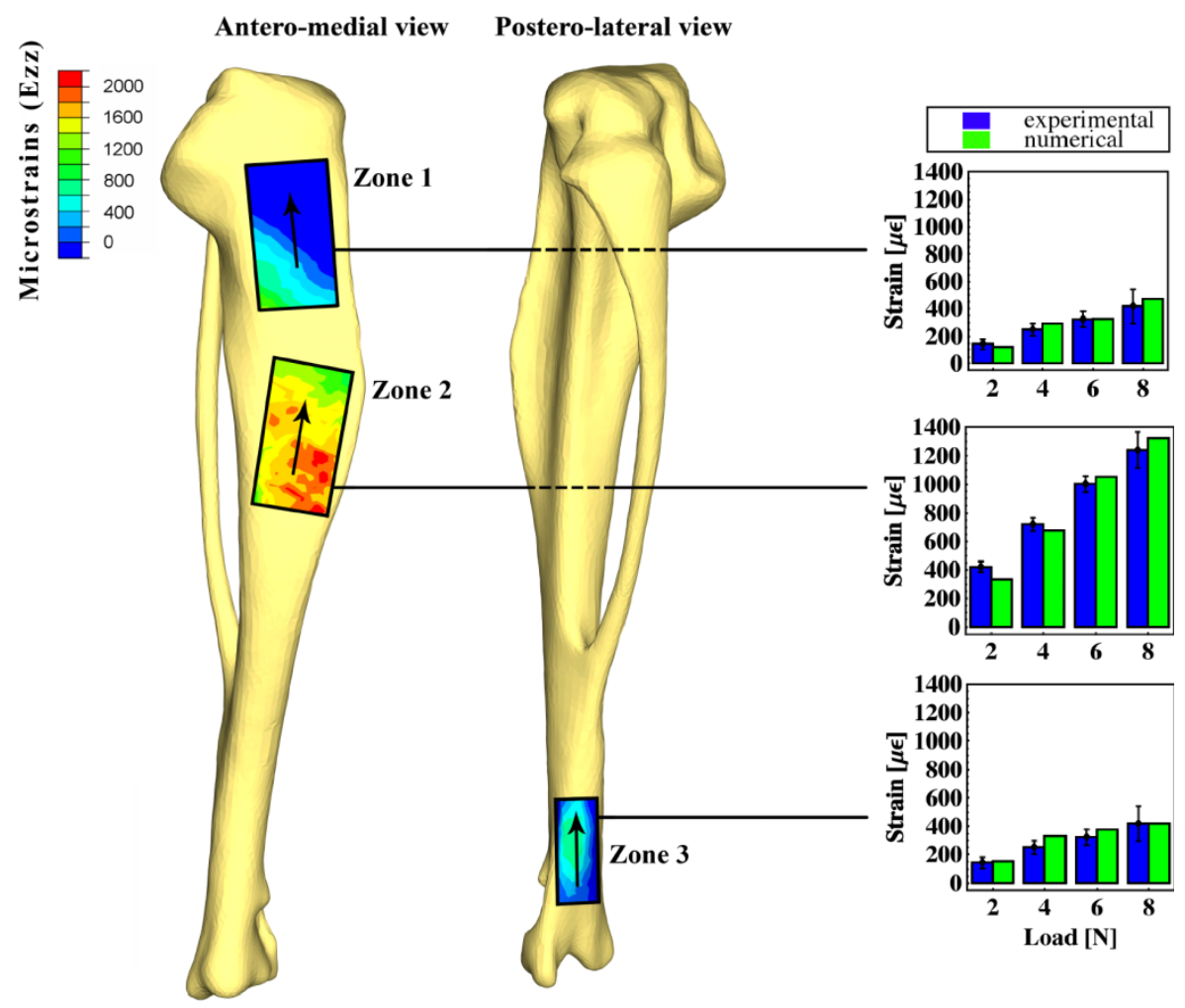

Figure 6. Distribution of the microstrains Ezz within each of the three measurement zones, relative to a locally defined coordinate system where the $z$-axis (arrows) is oriented along of the measurement axis and the $y$-axis is parallel to the measurement plate. Comparisons with experimental data are shown in the right column for each zone at different loading intensities. The relative difference are smaller than $10 \%$. Figure available in colour online. 


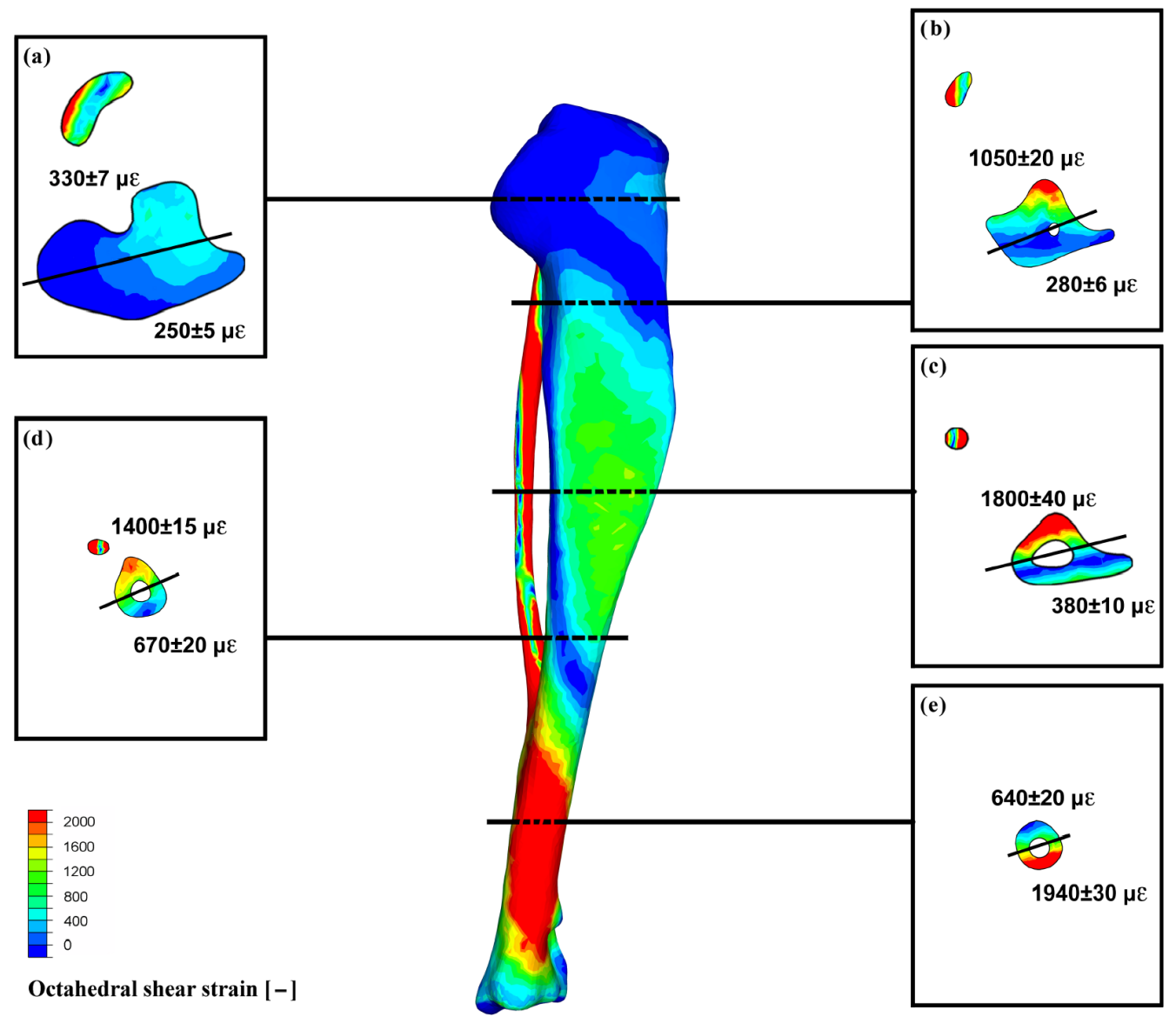

Figure 7. Octaedral shear strain distribution calculated numerically for five different locations: proximal tibia (a), proximal-diaphysis (b), tibial-crest diaphysis (c), midshaft (d) and distal tibia (e). Lines represent antero/postero separation and values represent the mean octaedral shear strain in the respective zone. Figure available in colour online.

can sense (Terrier et al. 1997). Thus, the 3D strain map will serve as a reference for our future histology and $\mu \mathrm{CT}$ planning, and it might as well be useful to any similar axial stimulation experiments.

To determine the 3D map of the octahedral shear strain distribution within the loaded tibia, a finite-element model of a mouse tibia was built from $\mu \mathrm{CT}$ images. The model was first validated with ex vivo experimental measurements of strain at three different locations on loaded tibias. Then the model was used to extrapolate these values to the entire tibial volume.

The maximal octahedral shear strain, calculated on the volumic model, was found at the postero-tibial crest $(1800 \pm 40 \mu \varepsilon)$ and the antero-distal tibia $(1940 \pm 30 \mu \varepsilon)$.
These results indicate that bone adaptation should be quantified in these areas, either for histological or histomorphological measurements, as a maximal mechanical stimulus will generate a maximal response of the tissue. It should be noted, however, than even in the maximal strain zone, differences in strain values exist inside the tibial section. A precise correlation between applied external mechanical stimulus and biological response can then be obtained only by using the combined approach (experimental/numerical) followed in this study.

The loading force-local strain relationship was shown to be linear in the three experimental zones until at least $1500 \mu \varepsilon$ strain. Using a comparable loading system, the experimental strain values are in accordance (max 6\%

Table 1. Mean \pm SEM octaedral shear strain $[\mu \varepsilon]$ for each orientation of each of the five zones.

\begin{tabular}{lccrrr}
\hline Orientation & Proximal tibia & Proximal diaphysis & Tibial crest & Midshaft tibia & Distal tibia \\
\hline Antero & $250 \pm 5$ & $280 \pm 6$ & $380 \pm 10$ & $670 \pm 20$ & $1940 \pm 30$ \\
Postero & $330 \pm 7$ & $1050 \pm 20$ & $1800 \pm 40$ & $1400 \pm 15$ & $640 \pm 20$ \\
\hline
\end{tabular}


difference) with De Souza et al. (2005). Our experimental set-up can then be considered as validated.

When experimental and numerical strain values were compared, a maximum difference of $10 \%$ was found for the three different zones. These differences can certainly be considered as acceptable, thus validating the numerical model. It should be mentioned that the results of the numerical model should be carefully considered for the very proximal and very distal part of the tibia, as the joint surface and the underlying trabecular bone might affect the numerical results. However, as the maximal strain is not found in these two regions, this aspect does not invalidate the determination of the maximal strain zone.

A limitation for the correlation process between external load and biological response is related to the fact that loads were applied in the axis of the tibia, inducing combined axial compressive and bending strains, due to the natural curvature of the bone. These different modes of strain were not accounted for in the presented analysis. Strictly from a biomechanical point of view, it is interesting to note that the numerical model indicated that the maximum bending was observed on the fibula. However, this information is of limited practical use as this bone is too small to place a gauge to confirm the numerical result. Moreover, this tissue is also too small for histology or histomorphometry analysis.

In this study, we assumed that the mechanical stimulus is directly related to strain. In the literature, different mechanical stimuli have been proposed to explain bone remodelling (Prendergast and Huiskes 1994; Parfitt 1996; Turner and Pavalko 1998; Huiskes et al. 2000). However, from an experimental point of view, only the tissue elongation transformed to a strain value is accessible. Other mechanical stimuli are mathematically related to the strain through constitutive laws. The calculated zone of highest strain obtained in this study can also be of general use for models using different descriptions of the mechanical stimulus.

To conclude, an experimental/numerical approach has been developed to identify the 3D octahedral shear strain map in an axial loading mode for a mouse tibia. It is proposed that quantification of bone adaptation should be performed at the tibial crest and at the distal diaphysis as the maximum biological response should correspond to the maximal applied strain. The numerical model could also be used to furnish a more subtle analysis as a precise correlation between local strain and local biological response can be obtained.

\section{Acknowledgements}

Project no. 04-P2 was supported by the AO Research Fund of the AO Foundation, Davos, Switzerland. We greatly acknowledge Marc Jeanneret for his technical support in designing and building the compression machine, Dr Kossi Agbeviade and André Badertscher for their support with micro strain-gages manipulation. We also thank Tyler Thacher for English editing.

\section{References}

Akhter MP, Cullen DM, Pedersen EA, Kimmel DB, Recker RR. 1998. Bone response to in vivo mechanical loading in two breeds of mice. Calcif Tissue Int. 63(5):442-449.

Carter DR. 1987. Mechanical loading history and skeletal biology. J Biomech. 20(11-12):1095-1109.

Chambers TJ, Evans M, Gardner TN, Turnersmith A, Chow JWM. 1993. Induction of bone-formation in rat tail vertebrae by mechanical loading. Bone Miner. 20(2): $167-178$.

Chow JW, Jagger CJ, Chambers TJ. 1993. Characterization of osteogenic response to mechanical stimulation in cancellous bone of rat caudal vertebrae. Am J Physiol. 265(2 Pt 1): E340-E347.

De Souza RL, Matsuura M, Eckstein F, Rawlinson SC, Lanyon LE, Pitsillides AA. 2005. Non-invasive axial loading of mouse tibiae increases cortical bone formation and modifies trabecular organization: a new model to study cortical and cancellous compartments in a single loaded element. Bone. 37(6):810-818.

Fritton JC, Myers ER, Wright TM, van der Meulen MCH. 2005. Loading induces site-specific increases in mineral content assessed by microcomputed tomography of the mouse tibia. Bone. 36(6):1030-1038.

Gardner MJ, van der Meulen MC, Demetrakopoulos D, Wright TM, Myers ER, Bostrom MP. 2006. In vivo cyclic axial compression affects bone healing in the mouse tibia. J Orthop Res. 24(8):1679-1686.

Huiskes R, Ruimerman R, van Lenthe GH, Jannsen JD. 2000. Effects of mechanical forces on maintenance and adaptation of form in trabecular bone. Nature. 405(6787):704-706.

Huiskes R, Weinans H, Grootenboer HJ, Dalstra M, Fudala B, Slooff TJ. 1987. Adaptive bone-remodeling theory applied to prosthetic-design analysis. J Biomech. 20(11-12): $1135-1150$.

Parfitt AM. 1996. Skeletal Heterogeneity and the purposes of Bone Remodeling. In: Marcus R, Feldman D, Kelsey JL, editors. Osteoporosis. San Diego (CA): Academic Press. p. 315-329.

Prendergast PJ, Huiskes R. 1994. Mathematical modeling of mircodamage in bone remodeling and adaptation. Bone Structure and Remodeling, Amsterdam.

Rho JY, Hobatho MC, Ashman RB. 1995. Relations of mechanical-properties to density and $\mathrm{Ct}$ numbers in human bone. Med Eng Phys. 17(5):347-355.

Rubin C, Gross T, Qin YX, Fritton S, Guilak F, McLeod K. 1996. Differentiation of the bone-tissue remodeling response to axial and torsional loading in the turkey ulna. J Bone Joint Surg Am. 78(10):1523-1533.

Rubin CT, Lanyon LE. 1984. Regulation of bone formation by applied dynamic loads. J Bone Joint Surg Am. 66(3): 397-402.

Terrier A, Rakotomanana RL, Ramaniraka AN, Leyvraz PF. 1997. Adaptation models of anisotropic bone. Comput Methods Biomech Biomed Eng. 1(1):47-59.

Turner CH, Pavalko FM. 1998. Mechanotransduction and functional response of the skeleton to physical stress: the mechanisms and mechanics of bone adaptation. J Orthop Sci. 3(6):346-355. 